\title{
Non-propagating electric and density structures formed through non-linear interaction of Alfvén waves
}

\author{
F. Mottez \\ Laboratoire Univers et Théories (LUTH), Observatoire de Paris; CNRS UMR8102, Université Paris Diderot, \\ 5 Place Jules Janssen, 92190 Meudon, France \\ Correspondence to: F. Mottez (fabrice.mottez@obspm.fr)
}

Received: 4 June 2011 - Revised: 13 September 2011 - Accepted: 5 December 2011 - Published: 9 January 2012

\begin{abstract}
In the auroral zone of the Earth, the electron acceleration by Alfvén waves is sometimes seen as a precursor of the non-propagating acceleration structures. In order to investigate how Alfvén waves could generate non-propagating electric fields, a series of simulations of counter-propagating waves in a homogeneous medium is presented. The waves propagate along the ambient magnetic field direction. It is shown that non-propagating electric fields are generated at the locus of the Alfvén waves crossing. These electric fields have a component orientated along the direction of the ambient magnetic field, and they generate a significant perturbation of the plasma density. The non-linear interaction of down and up-going Alfvén waves might be a cause of plasma density fluctuations (with gradients along the magnetic field) on a scale comparable to those of the Alfvén wavelengths. The present paper is mainly focused on the creation process of the non-propagating parallel electric field.
\end{abstract}

Keywords. Magnetospheric physics (Auroral phenomena) - Space plasma physics (Nonlinear phenomena; Numerical simulation studies)

\section{Introduction}

An auroral substorm is an abrupt increase in night-side hemispheric auroral power. The visual displays of the auroras happen at ionospheric altitudes (typically in the range 60$400 \mathrm{~km}$ ). A substantial part of the electron acceleration that triggers the auroras happens at a few thousands of kilometres above the ionosphere. Two main families of acceleration processes have been identified, associated to quasi-static electric fields, and to Alfvén wave electric fields. The acceleration by quasistatic, and basically non-propagating, electric fields (inverted $\mathrm{V}$, or other kinds of localized parallel electric fields) produces mono-energetic beams of electrons, while those by Alfvén waves are associated to broadband energy distribu- tions. Recent observations show that these two families of processes are not independent from each other.

A series of papers tend to conclude that quasi-static electric field structures might cause Alfvénic acceleration. An auroral plasma physics experiment on-board a rocket that flew into a $100 \mathrm{nT}$ auroral substorm explored an environment composed of an equatorward region of inverted $\mathrm{V}$ electron precipitation followed by a poleward region of mostly fieldaligned supra-thermal electron bursts. In the inverted $\mathrm{V}$ region (Klatt et al., 2005), the measured $\delta E / \delta B$ implied a stationary field-aligned current configuration. In the poleward region, the average electric field included time-varying components with periods of $0.5-5 \mathrm{~s}$, and $\delta E / \delta B$ was Alfvénic. The perpendicular scale size of most of these waves was on the order of a kilometer or less, as expected for acceleration by inertial Alfvén waves. The role of the Alfvénic acceleration in the poleward side of arcs was confirmed by conjugate observations from space and from the ground (Frey et al., 2010). It was proposed by Klatt et al. (2005) and others (Asamura et al., 2009; Chen et al., 2005), that the Alfvén waves might be triggered by the electron beam caused by the quasi-static electric fields. Then, the Alfvénic acceleration would be a consequence of the quasi-static electric structures.

Reciprocally, other papers suggest that Alfvénic processes might act as the precursors of quasi-static non-propagating acceleration structures. The optical observations reveal the development of a much brighter onset arc poleward of growth-phase arcs (Zou et al., 2010); the Alfvénic acceleration, mostly associated to the poleward side, might act as a precursor to the quasi-static electric fields. Newell et al. (2010) combined a very large number of identifications of substorm onsets from satellite global imagers with DMSP particle data to investigate the substorm cycle dependence of the diffuse aurora, monoenergetic aurora (produced by quasistatic electric fields), and broadband aurora (associated with Alfvénic acceleration). Although all types of aurora increase 
at substorm onset, they showed that broadband aurora are notably associated with substorms and, especially, substorm onset.

Evidences of acceleration structures emanating from Alfvén waves are also given by the direct observation of the parallel electric field (parallel to the mean direction of the magnetic field) (Chust et al., 1998; Chaston et al., 2007) or by the estimate of the wave Poynting flux (Louarn et al., 1994; Volwerk et al., 1996; Keiling et al., 2000). More references can be found in Keiling (2009). More recently, Hull et al. (2010) performed a case study of the development of an acceleration region based on multi-point Cluster observations in the high altitude auroral zone, across the plasma sheet and into the polar cap. They have identified an Alfvén wave dominated system, with its typical broad spectrum of accelerated particles, in the vicinity of a plasma cavity. After Alfvénic acceleration, the signature (the so-called inverted V) of particles accelerated by a quasi-static structure, such as a strong double layer, was observed. This suggests that the Alfvénic acceleration process could act as a precursor to the quasi-static process. But the Alfvén waves might not only be a cause for inverted-Vs. Ergun et al. (2005) have reported observations of spiky parallel electric fields associated to nonlinear Alfvén waves. Contrarily to inverted-Vs, these structures do not display any parallel electric current; they probably belong to an another category of electric field structures.

Considering the complexity of the auroral processes, it might be hazardous to state that one family of acceleration processes (non-propagating structures or waves) is systematically the consequence of the other. In the present study, without arguing against the possibility that quasi-static acceleration structures can generate Alfvén waves, we investigate the opposite case of quasi-static - or more precisely, nonpropagating - electric field acceleration structures triggered by Alfvén waves.

This paper presents a series of numerical simulations that investigate the interaction of down-going incident Alfvén waves with their up-going reflected counterpart. Their ability to create parallel stationary electric fields is questioned, as well as to prepare the auroral plasma before the setting of the acceleration processes. Because the duration of our simulation is short in comparison to the duration of the observed acceleration structures, one cannot conclude unambiguously on the quasi-static character of the structures generated through the Alfvén waves interaction, but we will state whether they are propagating or non-propagating. Complementary to the observational facts mentioned above, the approach in this paper is determined by a search for simplicity. That is why the initial conditions in the simulations are rather basic: we consider a plasma that is uniform in density, temperature, mean velocity and magnetic field. There is no mean current. In it, we superimpose two Alfvén waves, or two Alfvén wave packets, propagating along the ambient magnetic field direction $X$ (possibly in both directions), and we observe what happens, with special attention given to the possible emergence of a parallel electric field $E_{\mathrm{X}}$ and to the plasma density.

Because of this simple setting, the present study cannot pretend to bring any conclusion on the large scale structure of the auroral zone. Therefore, we cannot involve the large scale density and magnetic field dependence with altitude, net potential drops on large scales, etc. Because the numerical simulations are conducted with a reduced ion to electron mass ratio, quantitative comparisons with auroral data cannot be done yet. As well as the analysis of the accelerated particle distribution function and of the field aligned current, the quantitative analysis is left for a further stage of this investigation. This paper focuses on an explanation of the physical process observed in the simulations that are in the range of "microphysics" when compared to magnetospheric scales. Nevertheless, its potential relevance to auroral physics is the main motivation of this work.

It has been shown in previous studies (Hasegawa and Chen, 1975; Goertz, 1984) that, even in the context of linear theories, Alfvén waves with an oblique wave vector induce an electric field with a component parallel to the ambient magnetic field. This happens provided that the transverse scale is small enough to allow $k_{\perp} c / \omega_{\mathrm{p}} \sim 1$ or $k_{\perp} \rho_{\mathrm{i}} \sim 1$, where $k_{\perp}$ is the wave vector's component perpendicular to the ambient magnetic field, $\omega_{\mathrm{p}}$ is the plasma frequency, and $\rho_{\mathrm{i}}$ is the ion Larmor radius. In the present study, we do not suppose that the Alfvén waves are already accelerating the plasma. As the origin of their small transverse scales is not trivial, it is more careful, for the generality and the simplicity of the initial condition to neglect the presence of small transverse scale associated to the Alvfén waves. The choice of setting initially waves without small transverse scale also comes from the need to explain up-going waves. They should be the reflected part of down-going waves created in the magnetosphere, and reflected upon the ionosphere. Alfvén waves with small transverse scales of the order of $1 \mathrm{~km}$, like those associated to acceleration structures described by Klatt et al. (2005), cannot be reflected efficiently upon the ionosphere (Lessard and Knudsen, 2001). Therefore, we set initially waves that propagate along the magnetic field. Because the Alfvén waves in the present study propagate along the ambient magnetic field, the linear theory shows that they do not carry any parallel electric field, even when inertial and kinetic effects are considered. Therefore, the setting of a parallel electric field $E_{\mathrm{X}}$ presented in the next sections is the result of nonlinear effects.

Let us notice already that we make a distinction between the $X$ component of a vector (parallel to the ambient - and uniform - magnetic field) and its $x$ component, that is parallel to the local value of the magnetic field. The reason why is explained in Sect. 4. 


\section{Numerical method and simulation parameters}

As in Génot et al. (2000, 2001a, 2004), dedicated to the physics of auroral Alfvén waves, the numerical simulations are made with a EGC (Electron Guiding Center) electromagnetic PIC code that takes into account the motion of the electron guiding center and the full ion motion. The boundary conditions are periodic. A complete description of this code is given in Mottez et al. (1998).

The physical variables are reduced to dimensionless variables. Time and frequencies are normalized by the electron plasma frequency $\omega_{\mathrm{p} 0}$ that correspond to a reference background electron density $n_{0}$. Velocities are normalized to the speed of light $c$, and the magnetic field is given in terms of the dimensionless electron gyrofrequency $\omega_{\mathrm{ce}} / \omega_{\mathrm{p} 0}$. The mass unit is the electron mass $m_{\mathrm{e}}$. Therefore, the units (starting from the Maxwell equation in the MKSA system) are $c / \omega_{\mathrm{p} 0}$ for distances, $\omega_{\mathrm{p} 0} / c$ for wave vectors, $e$ for charges, $e n_{0}$ for the charge density, $c \omega_{\mathrm{ce}} / \omega_{\mathrm{p} 0}$ for the electric field. In the following parts of this paper, all the equations, numerical values and figures are expressed in this system of units.

This paper is based on a series of numerical simulations, whose naming convention is AWC (for Alfvén wave crossing) followed by a number, or a number and a letter. These simulations share most of their characteristics with AWC001, which is therefore used as a reference simulation. For the others, a few parameters are varied. When a physical parameter is varied (for instance, a wave amplitude), the number is different. When only computational parameters are varied (such as the number of macro-particles), a letter is added to the name of the simulation.

Here are some details about the parameters and initial values of AWC001. Two wave packets propagate in a uniform plasma, in opposite directions along the $\mathrm{X}$-axis. There are 819200 particles of each species in the simulation; it corresponds to 50 particles per cell. There are 2048 time steps, defined by $\Delta t=0.2$, corresponding to a total time $t_{\max }=409.6$. The size of the whole simulation domain is $4096 \Delta X \times 4 \Delta Y$, where $\Delta X=\Delta Y=0.1$ is the size of the grid cells. Therefore, the simulation is practically in one dimension. The electron thermal velocity is $v_{\text {te }}=0.1$; the ion and electron temperatures are the same. The ion to electron mass ratio is reduced to $m_{\mathrm{i}} / m_{\mathrm{e}}=400$.

Each wave packet is initialized as the sum of 8 sinusoidal waves with their maximum - in terms of magnetic perturbation - at $X_{1}=0$ for the first wave packet, and $X_{2}=241$ for the second wave packet. In each packet, the waves have the same amplitude, given by the wave magnetic field amplitude $\delta B=0.12$. The value of the background magnetic field is $B_{0}=4$. The waves have a right handed circular polarization. This corresponds, in parallel propagation, to the fast magneto-sonic branch of the dispersion relation. Their wavelengths are $\lambda=\lambda_{0} / m$ where $\lambda_{0}=409.6$ and $m$ varies from 1 to 8 . The phase velocities vary from 0.45 for the shortest wave to 0.23 for the longest. (The Alfvén velocity is $V_{\mathrm{A}}=0.2$.) The first wave packet propagates downward (toward positive values of $X$ ) and the second wave packet propagates upward (toward negative values of $X$ ).

For each of the eight sinusoidal waves (characterized by the mode number $m$ ), the wave vector $\boldsymbol{k}_{m}$ is parallel to the $\mathrm{X}$-direction, which is also the direction of the quasi-1-D box (with 4096 cells in the $\mathrm{x}$-direction and 4 cells in the $\mathrm{y}$-direction) and the direction of the ambient magnetic field $\boldsymbol{B}_{\mathbf{0}}=\left(B_{0}, 0,0\right)$.

Following a widespread convention, we call the direction of the wave vector the longitudinal direction. In the present case, it is given by the $\mathrm{X}$-axis. We say that the direction of the magnetic field is the parallel direction. With a wave of infinitesimal amplitude, the longitudinal and the parallel directions would coincide, but this it is not the case with finite amplitude Alfvén waves.

The polarization of each of these sinusoidal waves is given by initial values of the transverse components $B_{Y}(X)$, $B_{\mathrm{Z}}(X), E_{\mathrm{Y}}(X), E_{\mathrm{Z}}(X)$ of the electromagnetic field, and by the particles' velocity. Each macro-particle, electron ("e") or proton ("p"), of index " $i$ " has a random velocity, following a Gaussian law determined by the temperature, to which is added a transverse velocity perturbation $v_{\mathrm{eY}}\left(X_{i}\right), v_{\mathrm{eZ}}\left(X_{i}\right)$, $v_{\mathrm{pY}}\left(X_{i}\right), v_{\mathrm{pZ}}\left(X_{i}\right)$ that depends on the particle's position $X_{i}$ and nature (electron or proton). Each of these functions of $X$ is of the form $F(X)=\delta B \times f_{F m} \times \sin \left(k_{m}\left(X-X_{j}\right)\right)$ or $F(X)=\delta B \times f_{F m} \times \cos \left(k_{m}\left(X-X_{j}\right)\right)$, where $X_{j}$ is the position of the centre of the wave packet (one value for each wave packet), and $f_{F m}$ is a scalar that is different for every field $\left(B_{Y} \ldots v_{\mathrm{Z}}\right)$ and every mode $m$. For the magnetic field, $f_{B m}=1$. For the parallel electric field, $f_{\mathrm{Exm}}=0$. The other values of $f_{F m}$ are computed numerically, before the simulation, through the resolution of the linear dispersion equation of Alfvén waves in the cold bi-fluid approximation, as detailed in the appendix of Mottez (2008). At the center of a wave packet (represented by the values $X_{1}$ and $X_{2}$ for the first and the second wave packets), the amplitude of the magnetic field is initially $8 \times \delta B$, as the wave packet is the sum of 8 sinusoidal waves (labelled by their mode number $m$ varying from 1 to 8 ) with the amplitude $\delta B$.

This way of initializing Alfvén wave packets has already been applied and tested by Mottez and Génot (2011). It was shown that for a single wave packet propagating on a uniform plasma, the packet is not destroyed by the dispersion. Apart from noise at a frequency around the plasma frequency $(\omega \sim$ 1), for a moderate wave amplitude, still for a single wave packet, there is no creation of parallel electric field, or at least the electric field is below the noise level. This point will be discussed in the next section for waves of larger amplitude.

The parameters of the other simulations are presented in Table 1 . The initial values of the wave packets positions $X_{j}$ are varied in a few simulations in order to test any influence of phase and boundary conditions. Actually, none is seen. The values of $X_{j}$, of no influence, are not specified in Table 1. The simulations AWC001 to AWC010 are initialized 
Table 1. Simulation parameters varied from AWC001, and parallel electric field maximum value. Each column contains the data for the wave packets 1 and 2. RH (resp. LH) is for right-hand (resp. left-hand) polarized waves. D and U mean Downward and Upward propagation. The simulations AWC001 to AWC009 correspond to wave packets, each packet being the sum of 8 sinusoidal waves with the same amplitude. This amplitude, given in the column $\delta B$, can be different for the two wave packets. The range of wavelengths is indicated in the column $\lambda$. In the simulation AWC010-AWC019, there are no wave packets, but only two sinusoidal waves, whose wavelengths are given in the column $\lambda$. Versions "b" are made with more particles. Version "c" is a 2-D simulation (see text). In the last column is displayed the maximum amplitude of the parallel electric field at any place and any time in the simulation. The maximum intensity is reached at the locus of the wave packets interactions, except for AWC009, where the most intense value of $E_{\mathrm{X}}$ is associated to plasma wave oscillations. For AWC009, the second number indicates the amplitude associated with the Alfvén waves interaction.

\begin{tabular}{lcccccc}
\hline name & $N /$ cell & $\delta B$ & polar. & dir. & $\lambda$ & $\max \left(E_{X}(X, t)\right)$ \\
\hline wave packet & & & & & & \\
\hline AWC001 & 50 & 0.120 .12 & RH RH & D U & $51.2-409.51 .2-409$. & 0.12 \\
\hline AWC002+b & 50,400 & 0.080 .08 & LH LH & D U & $51.2-409.51 .2-409$. & 0.078 \\
AWC003+b & 50,400 & 0.080 .08 & RH LH & D D & $51.2-409.51 .2-409$. & 0.021 \\
\hline AWC004 & 50 & 0.160 .16 & RH RH & D U & $51.2-409.51 .2-409$. & 0.21 \\
AWC005 & 400 & 0.040 .04 & RH RH & D U & $51.2-409.51 .2-409$. & 0.016 \\
AWC006 & 400 & 0.020 .02 & RH RH & D U & $51.2-409.51 .2-409$. & 0.008 \\
AWC007 & 400 & 0.080 .04 & RH RH & D U & $51.2-409.51 .2-409$. & 0.030 \\
AWC008 & 400 & 0.080 .16 & RH RH & D U & $51.2-409.51 .2-409$. & 0.11 \\
\hline AWC009 & 400 & 0.080 .16 & RH RH & D D & $51.2-409.51 .2-409$. & 0.13 at $\omega_{\text {pe }}$
\end{tabular}

\begin{tabular}{lcclll}
\hline sinusoid & \multicolumn{5}{l}{} \\
\hline AWC010+b & $50,400(\mathrm{~b})$ & 0.080 .08 & RH RH & D U & 102.4102 .4 \\
\hline AWC010c, in 2-D & 40 & 0.080 .08 & RH RH & D U & 102.4102 .4 \\
\hline AWC011 & $50(b)$ & 0.080 .08 & LH RH & D U & 102.4102 .4 \\
AWC012 & $50,400(b)$ & 0.080 .08 & LH LH & D U & 102.4102 .4 \\
\hline AWC013+b & $50,400(b)$ & 0.160 .16 & RH RH & D U & 102.4102 .4 \\
AWC014+b & $50,400(b)$ & 0.040 .04 & RH RH & D U & 102.4102 .4 \\
AWC015+b & $50,400(b)$ & 0.080 .04 & RH RH & D U & 102.4102 .4 \\
AWC016+b & $50,400(b)$ & 0.080 .16 & RH RH & D U & 102.4102 .4 \\
AWC017+b & $50,400(b)$ & 0.040 .16 & RH RH & D U & 102.4102 .4 \\
AWC018+b & $50,400(b)$ & 0.020 .16 & RH RH & D U & 102.4102 .4 \\
AWC019+b & $50,400(b)$ & 0.020 .04 & RH RH & D U & 102.4102 .4 \\
\hline AWC020+b & $50,400(b)$ & 0.080 .08 & RH RH & D U & 409.6409 .6 \\
\hline
\end{tabular}

with two wave packets. The simulations after AWC010 are initialized more simply with only two sinusoidal waves propagating in opposite directions. As we will see, this simpler setting is a good help to better characterize the nature of their interaction. In simulations AWC13 to AWC019, only the amplitudes of the two waves are varied. Actually, these simulations have been done in two versions. The fist corresponds to 50 particles per cell, as in AWC001-AWC009. Other series (AWC010b and AWC013b-AWC019b) have been conducted with 400 particles per cell. These simulations "b", apart from being less noisy, provide the same results and the same numerical values (as far as the signal, and not the noise above it are concerned). The simulation AWC010 has also a version "c", made with a bi-dimensional grid of size
$2048 \times 1024,40$ particles per cell, and 512 time steps. The fields computed in this simulation show the same behaviour as in the 1-D simulations, and they remain invariant in the $y$-direction (as were the initial conditions), proving that the phenomenon under study is basically mono-dimensional.

A series of values of plasma parameters corresponding to the initial condition of IWC001, ICW002, ICW014 and ICW020 is presented, in ascending order, in Table 2. The ordering is consistent with the low density regions of the auroral zone, but because of the reduced ion to electron mass ratio, the values are closer to each other than in a real plasma. We can notice that in ICW001, the wave velocity is quite high compared to the MHD Alfvén wave velocity $\left(V_{\mathrm{A}}=0.20\right)$. The small wavelengths components of the wave packet rather 
Table 2. Plasma characteristic values in the initial conditions of simulations ICW001, ICW002, ICW014, ICW20. For each categories (lengths, velocities, frequencies, magnetic field), the data is ordered from the smaller to the largest values. The parameters that are varied from one simulation to another are boldfaced. One can notice that the value of the ion cyclotron frequency is plotted in two lines because of different orderings in IWC001, IWC002, ICW014 and ICW020. The data about the wave is given for a single wave packet; the characteristics of the second wave packet are the same, apart from their opposite directions of propagation.

\begin{tabular}{|c|c|c|c|c|}
\hline Simulation name & ICW001 & ICW002 & ICW014 & ICW020 \\
\hline \multicolumn{5}{|l|}{ Lengths } \\
\hline Electron larmor radius $\rho_{\mathrm{e}}$ & 0.025 & 0.025 & 0.025 & 0.025 \\
\hline Debye length $\lambda_{D}$ & 0.1 & 0.1 & 0.1 & 0.1 \\
\hline Ion Larmor radius $\rho_{\mathrm{i}}$ & 5 & 5 & 5 & 5 \\
\hline Lowest wavelength in the wave packet $(m=8)$ & 51.2 & 51.2 & 102.4 & 409.6 \\
\hline Largest wavelength in the wave packet $(m=1)$ & 409.6 & 409.6 & 102.4 & 409.6 \\
\hline \multicolumn{5}{|l|}{ Velocities } \\
\hline Thermal ion velocity $v_{\mathrm{ti}}$ & 0.005 & 0.005 & 0.005 & 0.005 \\
\hline Thermal electron velocity $v_{\text {te }}$ & 0.100 & 0.100 & 0.100 & 0.100 \\
\hline Lowest phase velocity in the wave packet & $0.225(m=1)$ & $0.071(m=8)$ & 0.32 & 0.225 \\
\hline Largest wave velocity in the wave packet & $0.452(m=8)$ & $0.169(m=1)$ & 0.32 & 0.225 \\
\hline \multicolumn{5}{|l|}{ Frequencies } \\
\hline Ion cyclotron frequency $\omega_{\mathrm{ci}}$ & & & 0.0100 & \\
\hline Lowest freq. in the wave packet $\omega_{\min }(m=1)$ & 0.0034 & 0.0025 & 0.0200 & 0.0034 \\
\hline Ion cyclotron frequency $\omega_{\mathrm{ci}}$ & 0.0100 & & & \\
\hline Largest freq. in the wave packet $\omega_{\max }(m=8)$ & 0.0556 & 0.0087 & 0.0200 & 0.0034 \\
\hline Ion cyclotron frequency $\omega_{\mathrm{ci}}$ & & 0.0100 & & 0.0100 \\
\hline Plasma frequency $\omega_{\mathrm{pe}}$ & 1 & 1 & 1 & 1 \\
\hline Electron cyclotron frequency $\omega_{\mathrm{ce}}$ & 4 & 4 & 4 & 4 \\
\hline \multicolumn{5}{|l|}{ Magnetic field } \\
\hline Maximum wave amplitude $\delta B_{\max }$ & 0.96 & 0.64 & 0.04 & 0.08 \\
\hline Ambient magnetic field $B_{0}$ & 4 & 4 & 4 & 4 \\
\hline
\end{tabular}

belong to the high frequency part of the dispersion relation branch. The simulation ICW002 has been conducted with two left-hand polarized waves. These waves' frequencies (from $\omega=0.0087$ for $m=8$ to $\omega=0.0025$ for $m=1$ ) are all lower than the ion gyrofrequency $\omega_{\mathrm{ci}}=0.01$ ), although still fairly close. We are clearly in the high frequency range of the Alfvén wave branches, like Electro Magnetic Ion Cyclotron waves (EMIC), also encountered in the auroral zone. With the simulation ICW020, the two waves are simple sinusoids, their phase velocity $V_{\Phi}=0.22$ is close to the MHD Alfvén velocity $V_{\mathrm{A}}=0.20$, and their frequency $\omega=0.0034$ is a third of the ion cyclotron frequency $\omega_{\mathrm{ci}}=0.01$. They correspond better to the characteristics of a MHD-like Alfvén wave.

In IWC001, the total wave amplitude magnetic field reaches $25 \%$ of the background field. This high wave amplitude is never met in the auroral zone. ICW001 is clearly a caricature in the sense that the plasma characteristics are exaggerated. The counterpart to this excess is a numerical simulations that could be conducted rather quickly, with a high signal/noise ratio that allows for an easier analysis of the physical processes at work. The other numerical simulations are conducted with wave intensities that are systematically lower than those in ICW001. For instance, in ICW014, both waves' amplitudes (here, simple sinusoids) represent $1 \%$ of the background magnetic field amplitude, a value more conform to those met in the high altitude auroral zone.

\section{Electrostatic field at the crossing of two Alfvén wave packets}

Figure 1 shows the magnetic field component $B_{\mathrm{Z}}(X, t)$ in simulation AWC001. We can clearly see two structures that propagate in opposite directions, with a moderate distortion, until they meet, around time 200. These structures, initially located around $X_{1}=0$ and $X_{2}=241$, are the two wave packets. They propagate with a uniform velocity and they are modified but not destroyed after their mutual interaction. Figure 2 shows the longitudinal electric field $E_{X}(X, t)$ in the same simulation. This electric field component is parallel to the wave vector and to the ambient magnetic field. We can see high frequency oscillations that do not propagate; they are simply a noise background oscillating around the 


\section{BZ}
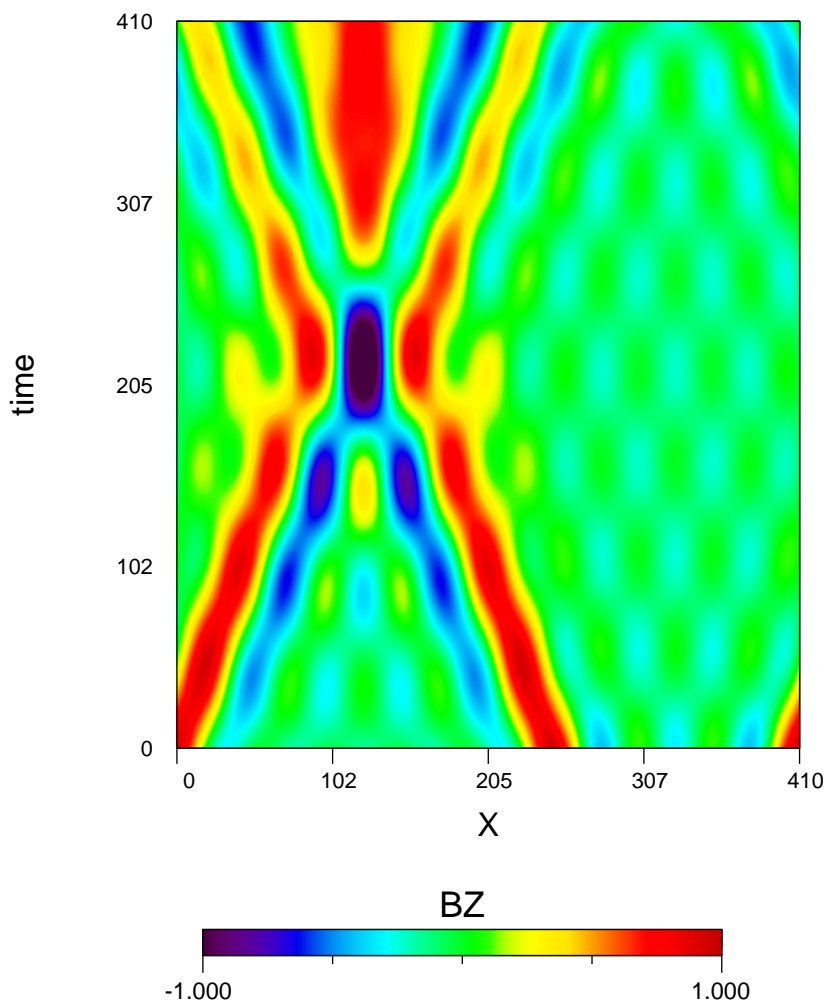

Fig. 1. AWC001(two wave packets). Temporal stack plot of the transverse magnetic field $B_{\mathrm{Z}}(X, t)$.

plasma frequency $(\omega=1)$. As in Mottez and Génot (2011), this noise is enhanced at the initial position of the wave packets because of an imperfect initialization of the Alfvén wave modes. More interesting, we can see that around time 200, when the two wave packets strongly interact, there is the emergence of an electric structure that does not propagate and that lasts as long as the two wave packets cross each other.

The simulation AWC002 is the interaction of two packets of shear Alfvén waves, characterized for parallel propagation, as left-hand circularly polarized waves. (The simulations AWC001 involve right-hand polarized waves.) Their phase and group velocity is smaller, and the simulation is conducted over a larger number of time steps (up to $t=$ 1200). Here again, when the two wave packets meet, a structured longitudinal electric field $E_{\mathrm{X}}$ appears. Nevertheless, the interaction of the two wave packets takes a time that is larger than those at which the simulation stops. It is possible that specific effects associated to the low velocity of the waves and maybe to ion giro-resonant effects play a supplementary role, not seen with right-hand circularly polarized waves.

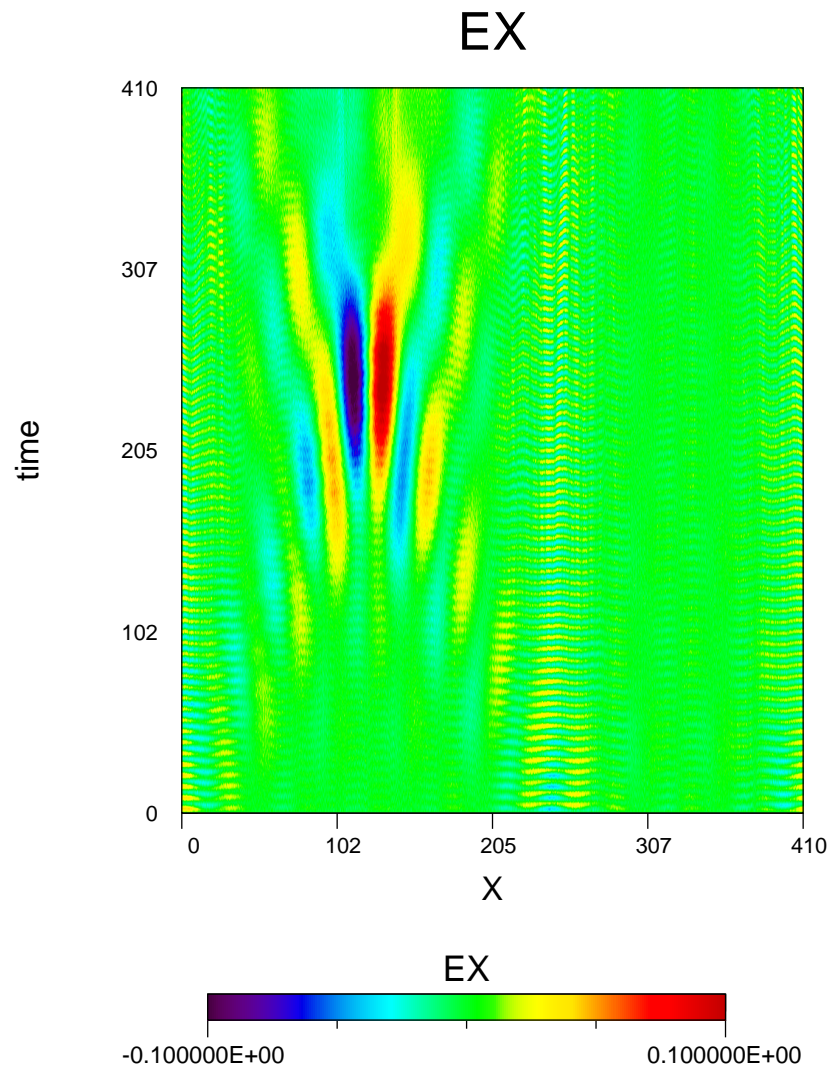

Fig. 2. AWC001(two wave packets). Temporal stack plot of the longitudinal electric field $E_{\mathrm{X}}(X, t)$.

The simulation AWC003 is based on the fact that a righthand side polarized packet propagates faster than a left handside polarized one. Therefore, when can test the interaction of two wave packets propagating in the same direction. Simulation AWC003 shows that when the faster wave packet reaches the slower one, a parallel electric field structure develops again, as shown on Fig. 3. Of course, in this circumstance, the electric field structure is propagating too, as the locus of the interaction between the two wave packets. Nevertheless, the parallel electric field reaches a level that is about 5 times smaller than in AWC002, in spite of Alfvén waves of the same amplitude. Therefore, the fact that the two waves are not counter-propagating may have an influence on the level of the parallel electric field.

The simulations AWC004-AWC008 test the conditions of AWC001 with different wave packets amplitudes and initial locations. As the signal is expected to be lower, because of smaller wave amplitudes, we have lowered the noise level by raising the number of macro-particles to 400 per cell. Again, a parallel electric field structure emerges, as in AWC001.

The last simulation of this series, AWC009 is the superposition of two neighbouring wave packets of high amplitude and propagating at the same velocity. Actually, it is similar to a very intense single wave packet and it appears that there 
EX
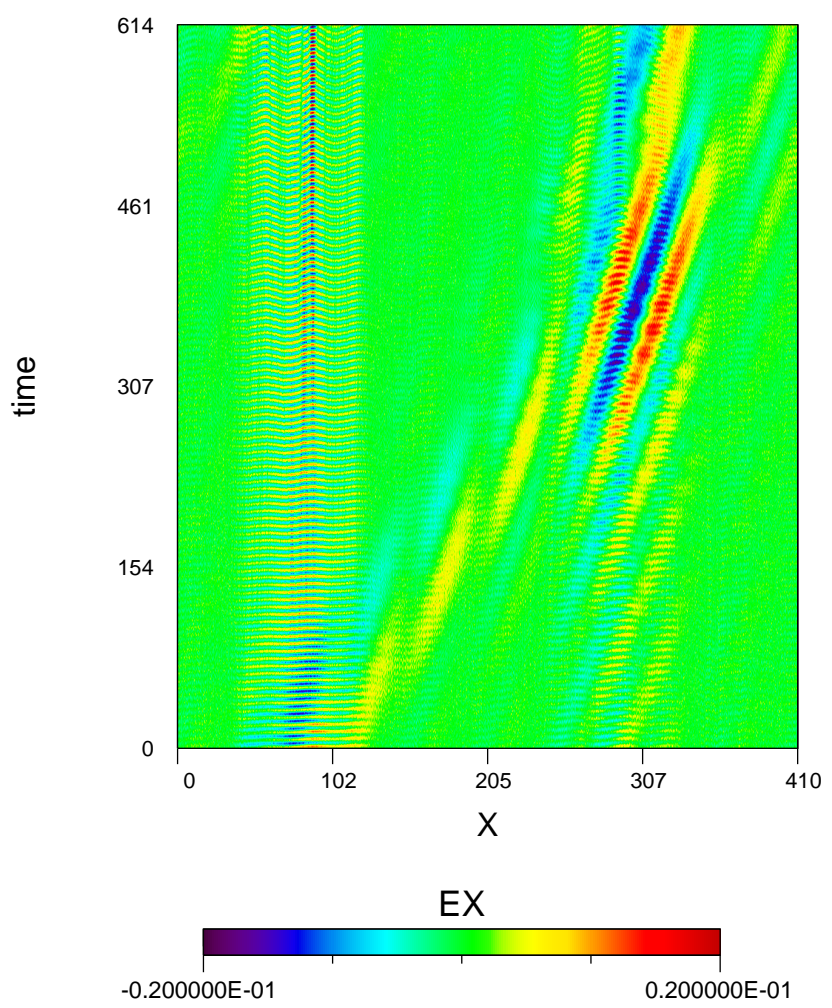

Fig. 3. AWC003 (a slower and a faster wave packets). Temporal stack plot of the longitudinal electric field $E_{X}(X, t)$.

is a longitudinal electric field $E_{\mathrm{X}}$ propagating with same velocity as the waves.

For a better understanding of what happens, a series of simulations with simpler initial conditions has been made. They consist of two sinusoidal counter-propagating waves instead of two wave packets.

Figure 4 shows the magnetic field component $B_{\mathrm{Z}}(X, t)$ in simulation AWC010. The space time dependency of the amplitude results from the superposition of the two counter propagating waves of equal velocity modulus and amplitudes. We can see that there are four wavelengths along the simulation box (the initially selected mode number is $m=4$ ). Figure 5 shows the corresponding electric field $E_{\mathrm{X}}$.

We can see that there is a sinusoidal dependency of mode $m=8$ and of null frequency. In order to confirm this observation, we have made space-time Fourier transforms of $B_{\mathrm{Y}}(X, t), B_{\mathrm{Z}}(X, t), E_{\mathrm{X}}(X, t), E_{\mathrm{Y}}(X, t)$, etc. The Figs. 6 and 7 show the Fourier transforms of $B_{Z}$ and $E_{X}$. As the simulation box is treated with periodic boundary conditions, the space Fourier transform is clean. The time Fourier transforms is made with 1024 samples, covering the whole simulation. The time series was apodized in a simple way: the reversed time signal was appended to the direct one. As the total duration of the simulation is not a multiple of the

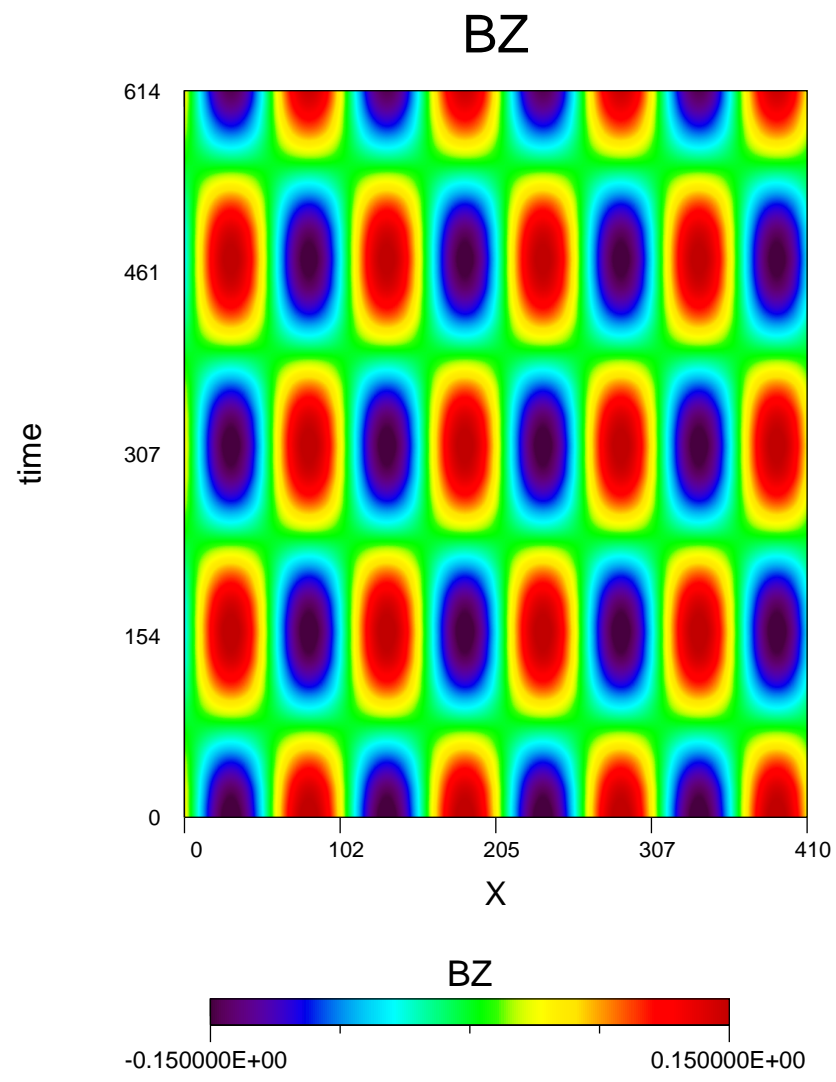

Fig. 4. AWC010 (two sinusoids). Temporal stack plot of the transverse magnetic field $B_{\mathrm{Z}}(X, t)$.

Alfvén wave frequency, there is a rather important aliasing effect. Nevertheless, it is possible to distinguish the time dependent modes from those that are constant (in the last case, there is no aliasing). We can see in Fig. 6 that the dominant amplitude is on the mode $m=4$, in accordance with the initial conditions, and that the frequency is non zero, with a peak that is compatible with the theoretical wave frequency of 0.02 . There is a second peak for the mode $m=12$ that is two orders of magnitude below, which is not analysed further in the present study. The Fig. 7 shows that for the $E_{X}$ electric field, the dominant mode corresponds to $m=8$ and to a null frequency. These Fourier transforms confirm what could be seen in Figs. 4 and 5. The Fourier analysis allows to go a bit further. Concerning $B_{\mathrm{Z}}$ (Fig. 6) and $B_{\mathrm{Y}}$ (not shown), there is no signal for $m=8$. The Fourier transforms of $E_{\mathrm{Y}}$ and $E_{\mathrm{Z}}$ show an intense peak for $m=4$ and a smaller for $m=8$.

These spectra suggest that the mode $m=8$ seen in the electric field is electrostatic, and that it is the result of nonlinear wave coupling between the two Alfvén waves. Indeed, let us call $\left(\omega_{\mathrm{A}}, k_{\mathrm{A}}\right)$ and $\left(-\omega_{\mathrm{A}}, k_{\mathrm{A}}\right)$ the frequencies and wave vector of the two counter-propagating Alfvén waves, where their coupling would result in the two modes $\left(\omega_{\mathrm{A}}, k_{\mathrm{A}}\right)+$ $\left(-\omega_{\mathrm{A}}, k_{\mathrm{A}}\right) \rightarrow\left(0,2 k_{\mathrm{A}}\right)$. 

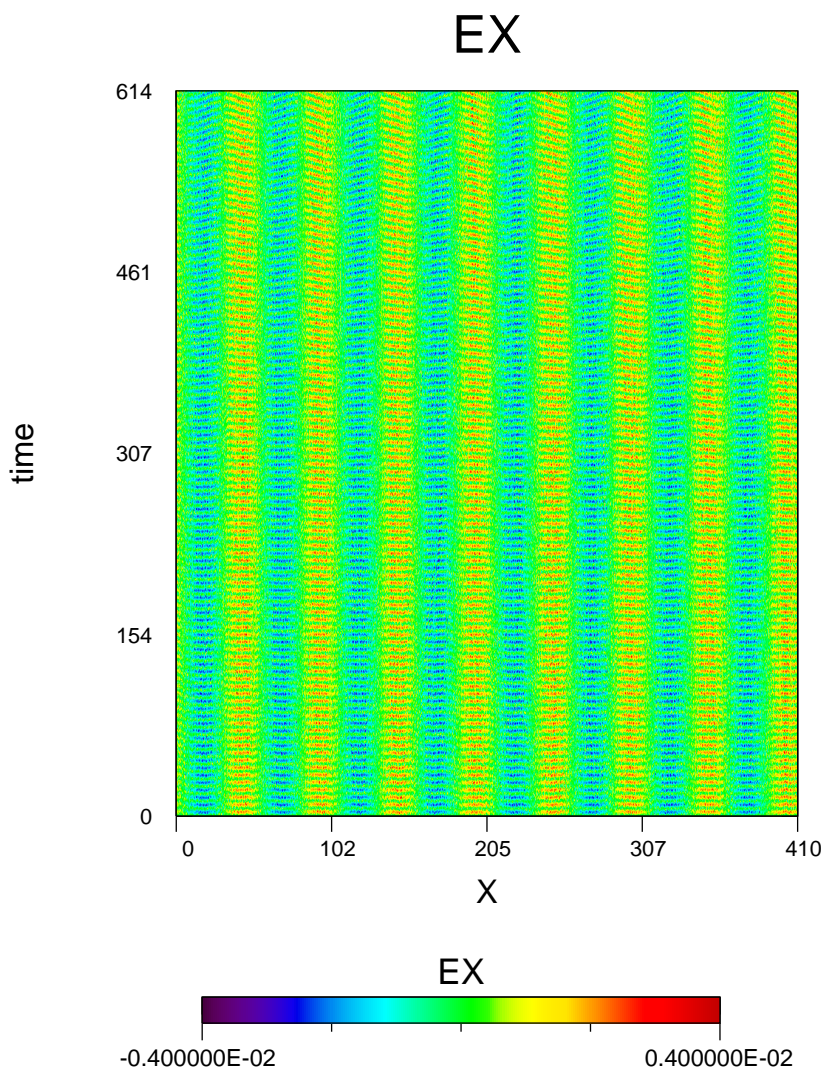

Fig. 5. AWC010 (two sinusoids). Temporal stack plot of the longitudinal electric field $E_{\mathrm{X}}(X, t)$.

As mentioned in Sect. 2, the simulations ICW001ICW019 involve Alfvén waves of quite high frequency, unlike the Alfvén waves met in the auroral zone. In order to study the influence of lower frequencies, ICW020 has been conducted with two sinusoidal waves with the same wavelength as the length of the simulation box. These waves have a velocity close to the MHD Alfvén wave velocity, and a frequency significantly lower than the ion gyrofrequency (see Table 2). This simulation also exhibits a purely electric wave with a component $E_{\mathrm{X}}$ of finite amplitude, showing the same characteristics as those described above. The maximum amplitude of $E_{\mathrm{X}}$ in ICW020 is 0.0036 . A similar value is attained in ICW010, made with two sinusoidal waves with the same amplitude but a four times shorter wavelength. Therefore, we can conclude that the emergence of the electric field component $E_{\mathrm{X}}$ is not a consequence of the rather high frequency of the wave on the Alfvénic branches used in the other simulations.

\section{Theoretical interpretation of the longitudinal electric field}

A full MHD approach of a large amplitude Alfvén wave, such as the simple wave described by Passot and Vázquez-
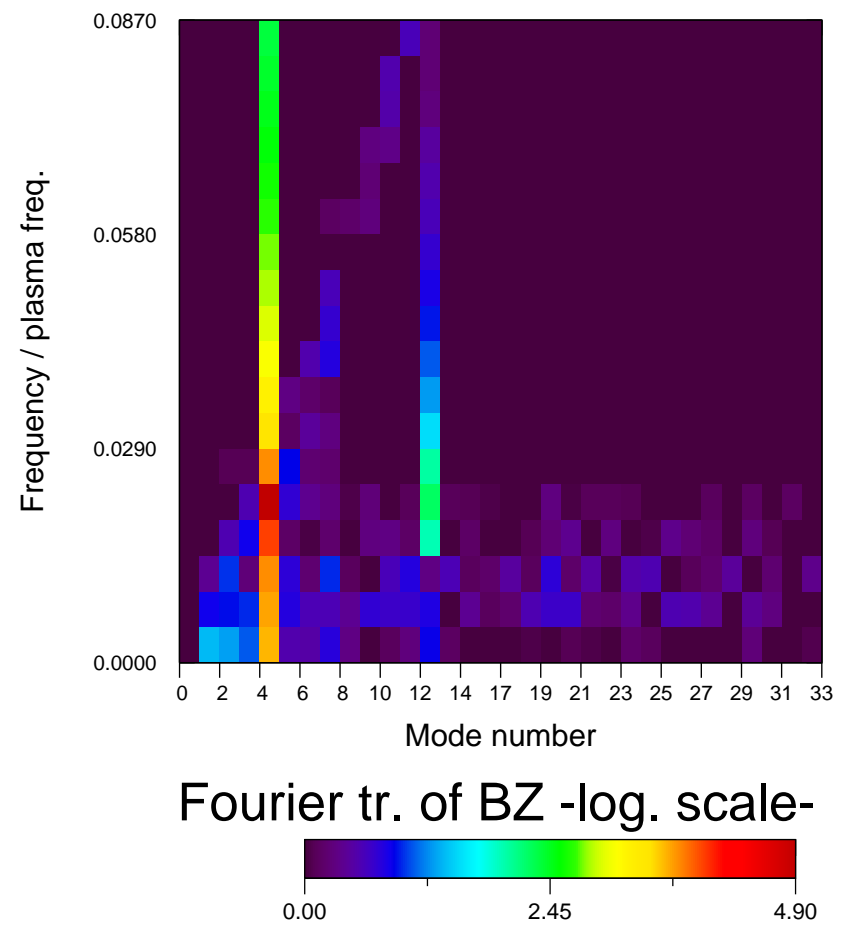

Fig. 6. AWC010 (two sinusoids). Space-time Fourier transform of $B_{\mathrm{Z}}(X, t)$. The horizontal axis displays the spatial mode number, and the vertical axis represents the frequency. The colour scale shows the logarithm of the mode amplitudes.

Semadeni (2003), shows that a single wave of finite amplitude in parallel propagation along the magnetic field can be indeed associated to a mass density perturbation. Due to the limitations of MHD, the charge density perturbation and the associated electric parallel field are not investigated by these authors.

An interpretation of parallel electric fields has been proposed by Tsiklauri (2006), in the context of parallel electric field generation by an Alfvén wave for an application to Sun coronal heating. It is based on the fact that $E_{\mathrm{X}}$ is not locally parallel to the magnetic field. At the location where the Alfvénic perturbation is high, the intensity of the $B_{\mathrm{Y}}, B_{\mathrm{Z}}$ magnetic field (1.7) reaches $40 \%$ of the ambient magnetic field $\left(B_{0}=4\right)$. Therefore, $X$ is not any more the local parallel direction to the magnetic field. This fact, resulting from the non-linearity of the waves, will be helpful in the further interpretation of the phenomenon.

Let $X, Y$ be the frame of the simulation, where $X$ (the longitudinal direction) is the direction of the wave vector $\boldsymbol{k}$ and the ambient magnetic field $B_{0}$. Because of the null divergence of $\boldsymbol{B}, B_{\mathrm{X}}=B_{0}$, and the magnetic perturbation has only Y- and Z-components. For simplicity, but without entailing the generality of the computation, we consider only the X-and Y-components of the vectors (considering the Z-component would not change the conclusions). For two counter-propagating waves, the magnetic perturbation is 


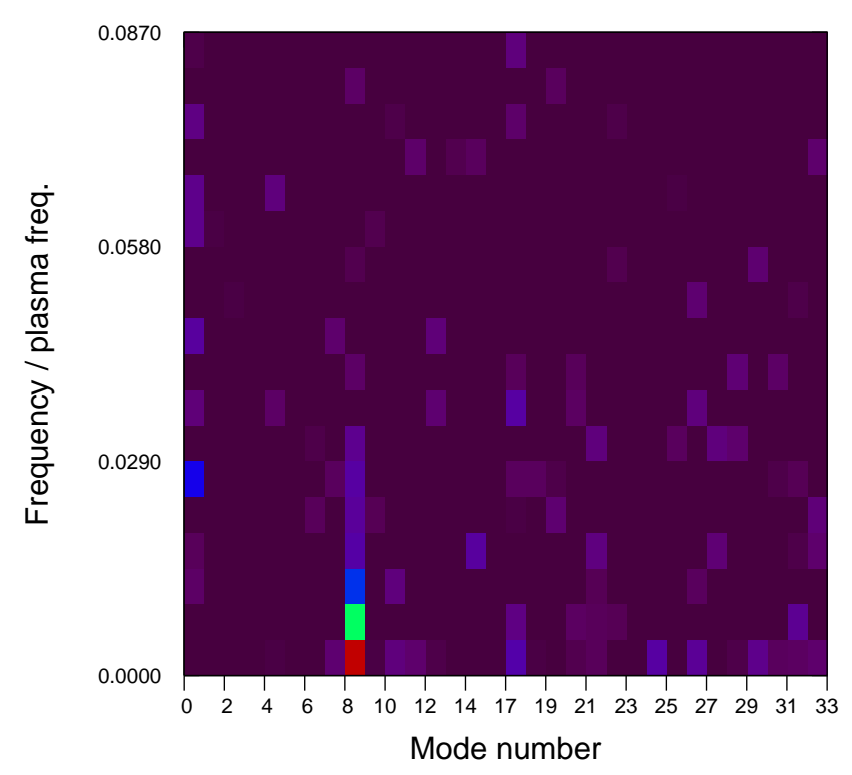

Fourier tr. of EX -log. scale-

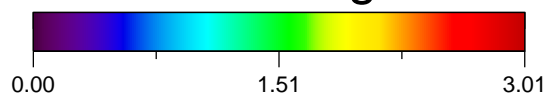

Fig. 7. AWC010 (two sinusoids). Space-time Fourier transform of $E_{X}(X, t)$. The horizontal axis displays the spatial mode number, and the vertical axis represents the frequency. The colour scale shows the logarithm of the mode amplitudes.

written as

$B_{\mathrm{Y}}(X, t)=B_{1} \cos \left(\omega t-k X+\phi_{B 1}\right)+B_{2} \cos \left(\omega t+k X+\phi_{B 2}\right)$.

Let $(x, y)$ (different from $X, Y)$ be the orthogonal coordinates, where $x$ is parallel to the local magnetic field. A magnetic field line, because of the perturbation by the waves, is given by

$\frac{d Y}{d X}=\frac{B_{Y}}{B_{X}}=\frac{B_{Y}}{B_{0}}$.

The condition $\boldsymbol{E} \cdot \boldsymbol{B}=0$ implies

$E_{\mathrm{X}}=E_{\mathrm{y}} \sin \theta$

where $\theta$ is the angle between the $\mathrm{X}$ - and the $\mathrm{x}$-axis, given by

$\sin \theta \sim \tan \theta=d Y / d X$.

As the electric field is perpendicular to the magnetic field, $E_{\mathrm{x}}=0$ and $E_{\mathrm{y}}$ can be written as

$E_{\mathrm{y}}=E_{1} \cos \left(\omega t-k X+\phi_{E 1}\right)+E_{2} \cos \left(\omega t+k X+\phi_{E 2}\right)$.

The coefficients $\phi_{B 1}, \phi_{B 2}, \phi_{E 1}$, and $\phi_{E 2}$ are appropriate numbers, independent from location and time, determined by the waves polarizations. From the above equations, the longitudinal electric field becomes

$$
\begin{aligned}
E_{X} & =\frac{E_{1} B_{1}}{2 B_{0}} \cos \left(\phi_{B 1}-\phi_{E 1}\right) \\
& +\frac{E_{2} B_{2}}{2 B_{0}} \cos \left(\phi_{B 2}-\phi_{E 2}\right) \\
& +\frac{E_{1} B_{1}}{2 B_{0}} \cos \left(2 \omega t-2 k X+\phi_{B 1}+\phi_{E 1}\right) \\
& +\frac{E_{2} B_{2}}{2 B_{0}} \cos \left(2 \omega t+2 k X+\phi_{B 2}+\phi_{E 2}\right) \\
& +\frac{E_{1} B_{2}}{2 B_{0}} \cos \left(2 \omega t+\phi_{B 2}+\phi_{E 1}\right) \\
& +\frac{E_{2} B_{1}}{2 B_{0}} \cos \left(2 \omega t+\phi_{B 1}+\phi_{E 2}\right) \\
& +\frac{E_{1} B_{2}}{2 B_{0}} \cos \left(+2 k X+\phi_{B 2}-\phi_{E 1}\right) \\
& +\frac{E_{2} B_{1}}{2 B_{0}} \cos \left(-2 k X+\phi_{B 1}-\phi_{E 2}\right)
\end{aligned}
$$

For a circularly polarized wave, the Faraday law implies that $\phi_{B 1}-\phi_{E 1}= \pm \pi / 2$ and $\phi_{B 2}-\phi_{E 2}= \pm \pi / 2$. Therefore, the two first terms of the above equation cancel.

With only one wave of mode $m$, this equation reduces to

$E_{X}=\frac{E_{1} B_{1}}{2 B_{0}} \cos \left(2 \omega t-2 k X+\phi_{B 1}+\phi_{E 1}\right)$

and the $E_{X}$ perturbation has a wave vector $2 k$ (mode $2 m$ ) that propagates with the same velocity as the Alfvén wave.

With two waves, we can see the appearance of two other kinds of signal. The first is a stationary wave of mode $2 m$, which corresponds exactly to what is seen on the simulations AWC010 and AWC011-AWC020. The second is a wave of infinite length and a frequency $2 \omega$.

We can notice that, according to this explanation, the setting of the $E_{\mathrm{X}}$ electric field is not progressive, but quasiinstantaneous, as seen in our simulations.

Here is a derivation of $E_{\mathrm{X}}$ in the particular case of two counter-streaming circularly right-handed waves. The wave coefficients, given in a fully developed explicit form in Mottez (2008), are such that, setting arbitrarily the value of $\phi_{E 1}$,

$$
\begin{gathered}
\phi_{E 1}=0 \text { and } \phi_{B 1}=-\frac{\pi}{2} \\
\phi_{E 2}=\phi_{0} \text { and } \phi_{B 2}=\phi_{0}+\frac{\pi}{2},
\end{gathered}
$$

where $\phi_{0}$ represents the phase shift between the wave 1 and the wave 2 . As the two waves have the same polarization, $E_{1} B_{2}=B_{1} E_{2}$. Inserting these values in Eq. (6), one finds

$$
\begin{aligned}
E_{X}= & +\frac{E_{1} B_{1}}{2 B_{0}} \cos \left(2 \omega t-2 k X-\frac{\pi}{2}\right) \\
& +\frac{E_{2} B_{2}}{2 B_{0}} \cos \left(2 \omega t+2 k X+2 \phi_{0}+\frac{\pi}{2}\right) \\
& +\frac{E_{1} B_{2}}{B_{0}} \cos \left(2 k X+\phi_{0}+\frac{\pi}{2}\right)
\end{aligned}
$$


Table 3. Test of the bi-linear dependency of the maximal Fourier transform value $\tilde{E}_{\mathrm{X}}(k)$ of $E_{\mathrm{X}}$ as a function of the initial Alfvén waves amplitudes. The initial Alfvén wave amplitudes $\delta B_{1}$ and $\delta B_{2}$ are given in the column $\delta B$. For a bilinear dependency, the factor $Q=\tilde{E}_{0 ., 8} / \delta B_{1} \times \delta B_{2}$ must be constant. In the column $\tilde{E}_{\mathrm{X}}(0 ., 8)$, the values in parenthesis correspond to simulations with 50 particles per cell, the other values correspond to the simulations with 400 particles per cell. When there is no difference, only one value is given.

\begin{tabular}{lcll}
\hline name & $\delta B$ & $\tilde{E}_{\mathrm{X}}(0 ., 8)$ & $Q$ \\
\hline AWC010 & 0.080 .08 & $502 .(505)$. & 1.00 \\
AWC013 & 0.160 .16 & 1953. & 0.972 \\
AWC014 & 0.040 .04 & 127. & 1.001 \\
AWC015 & 0.080 .04 & $252 .(253)$. & 1.003 \\
AWC016 & 0.080 .16 & 994. & 0.990 \\
AWC017 & 0.040 .16 & $498 .(500)$. & 0.992 \\
AWC018 & 0.020 .16 & 250. & 0.996 \\
AWC019 & 0.020 .04 & 64. (62.6) & 1.01 \\
AWC020 & 0.080 .08 & $376 .(377)$. & 0.749 \\
\hline
\end{tabular}

The two terms corresponding to a wave of infinite length cancel (phase opposition), and those corresponding to the time invariant signal, in phase, add to each other, making the time invariant electric field the dominant term.

In the particular case of two counter-streaming circularly waves of opposite circular polarisation,

$$
\begin{gathered}
\phi_{E 1}=0 \text { and } \phi_{B 1}=+\frac{\pi}{2} \\
\phi_{E 2}=\phi_{0} \text { and } \phi_{B 2}=\phi_{0}+\frac{\pi}{2},
\end{gathered}
$$

and

$$
\begin{aligned}
E_{X}= & +\frac{E_{1} B_{1}}{2 B_{0}} \cos \left(2 \omega t-2 k X-\frac{\pi}{2}\right) \\
& +\frac{E_{2} B_{2}}{2 B_{0}} \cos \left(2 \omega t+2 k X+2 \phi_{0}+\frac{\pi}{2}\right) \\
& +\frac{E_{1} B_{2}}{B_{0}} \cos \left(2 \omega t+\phi_{0}+\frac{\pi}{2}\right) .
\end{aligned}
$$

In that case, the coupling term between the two waves (the one proportional to $E_{1} B_{2}$ ) is purely time dependent, as if for a wave of infinite wavelength.

The next section is devoted to a comparison of this simple theoretical model with the simulation results.

\section{Testing of the properties of the longitudinal electric field}

This coupling process is typically 1-D, and it should happen in the same way in a 2-D simulation with the same initial conditions. This is what has been checked with the simulation AWC010c, made on a box of size $4096 \times 1024$ (instead of $4096 \times 4$ for the other simulations). The behaviour of the

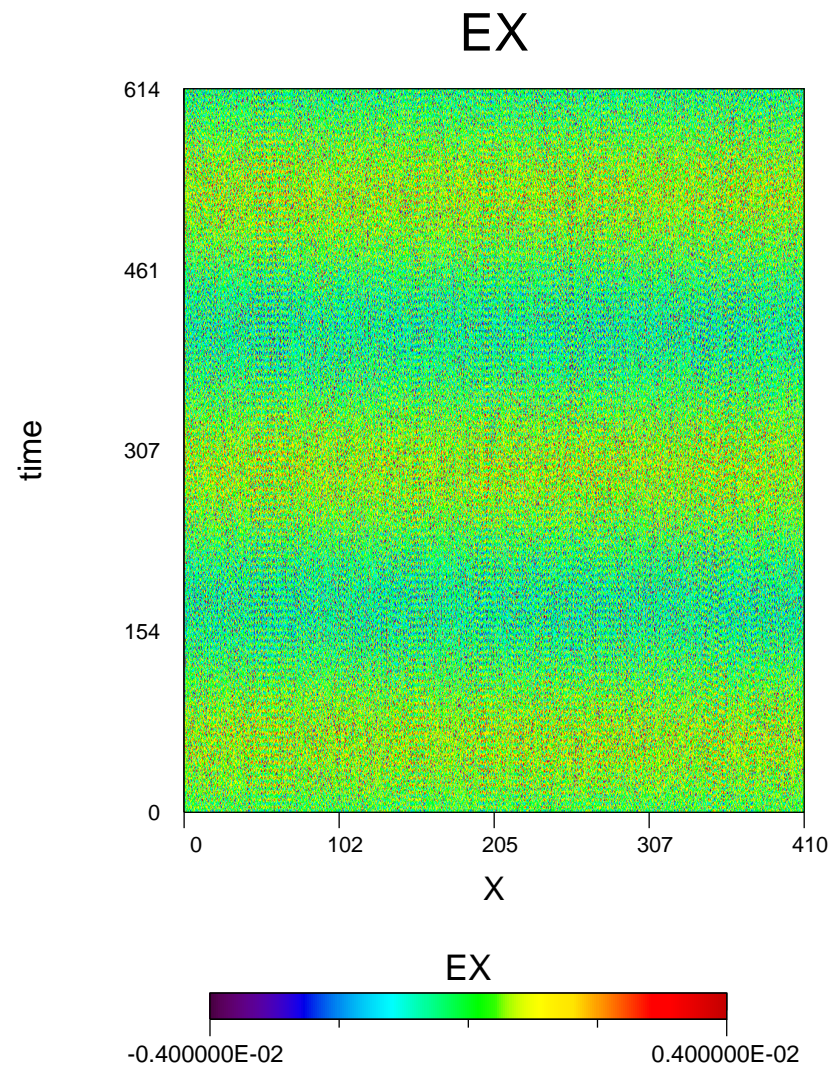

Fig. 8. AWC011. Temporal stack plot of the longitudinal electric field $E_{\mathrm{X}}(X, t)$.

plasma in this simulation is the same as in 1-D, and the fields remain invariant in the y-direction.

The simulation AWC011 shows the interaction of a lefthand polarized wave crossing a right hand polarized one. The electric field is displayed in Fig. 8. We can see a mode of zero wave number and a frequency that is the double of the Alfvén waves frequency. This is compatible with the other possible wave coupling, which consists of the difference of the two initial Alfvén waves, considered as two pump waves, $\left(\omega_{\mathrm{A}}, k_{\mathrm{A}}\right)-\left(-\omega_{\mathrm{A}}, k_{\mathrm{A}}\right) \rightarrow\left(2 \omega_{\mathrm{A}}, 0\right)$, whose resulting electric field $E_{X}$ is given by the wave coulpling term in Eq. (13).

According to Eq. (6), the amplitude dependence must be quadratic, or more precisely, linearly dependent on the amplitude of each of the two pump/Alfvén waves. In order to check this, the AWC012-AWC019 simulations have been conducted. The characteristics of the initial Alfvén waves are the same as in AWC010, except for their amplitude that take various values. In all cases, an electric field similar to those in AWC010 is observed. One expects that the amplitude of the mode $\omega=0 ., m=8$ in the Fourier transform (we use the notation $\left.\tilde{E}_{0 ., 8}\right)$ is proportional to the product $\delta B_{1} \times \delta B_{2}$ of the two Alfvén waves. Therefore, a ratio $Q=\tilde{E}_{0 ., 8} / \delta B_{1} \times \delta B_{2}$ has been computed. It is displayed in Table 3 . We can check that $Q$ is constant, within a margin of one percent. 
The Fourier transforms displayed in Fig. 7 show indeed that the term of null frequency and $2 k$ wave vector is dominant in the expression of $E_{X}$, as seen in Eq. (10). But the two first terms of this equation are not supposed to be null; nevertheless, they do not appear in the spectrum of Fig. 7. Two other simulations with the same parameters but different values of $\phi_{0}$ have been performed, and they show the same behaviour. A similar remark can be done about the simulation AWC009... and all the others. This is not explained by the little theory exposed in Sect. 4. The theory given in Sect. 4 would correspond to the simulations if only the terms that couple two different waves would be kept. It would mean that the different waves couple to each other, but that they do not couple to themselves. This might be the case if the single sinusoidal solution of the linear wave equation would also be a solution of the nonlinear equations, as for ideal MHD Alfvén waves. If we make this supposition, the only term remaining in Eq. (10) would be simply

$$
E_{\mathrm{X}}=\frac{E_{1} B_{2}}{B_{0}} \cos \left(2 k X+\phi_{0}+\frac{\pi}{2}\right)
$$

and this would correspond to what is seen with the simulation AWC010 on Fig. 5, where the electric field $E_{\mathrm{X}}$ has no time dependence. Similarly, the only term remaining in Eq. (13) would be

$E_{\mathrm{X}}=\frac{E_{1} B_{2}}{B_{0}} \cos \left(2 \omega t+\phi_{0}+\frac{\pi}{2}\right)$.

Again, this would correspond to the case of AWC011, where, as seen on Fig. 8, the electric field $E_{\mathrm{X}}$ is purely time dependent.

\section{A glimpse at slower processes}

The simulations conducted with two input Alfvén waves show that the electric field $E_{\mathrm{X}}$ (whatever sinusoidaly time or space dependent) reaches a constant amplitude quasiinstantaneously. Does it mean that there is no slower evolution of the plasma properties? No. Figure 9 shows a stack plot of the electron density $n_{\mathrm{e}}(X, t)$. We can see that it has an increasing modulation associated to the same wave-mode ( $m=8)$ as the longitudinal electric field $E_{\mathrm{X}}$. There is obviously a link between this amplification of the density modulation and the particle acceleration by the parallel electric field $E_{\mathrm{X}}$. But at the same time, a part of the process maybe connected also to the wave coupling process. Actually, no amplification is seen either on the perpendicular electric field components $E_{\mathrm{Y}}$ and $E_{\mathrm{Z}}$, and there is no obvious amplification either of the charge density (compatible with a constant value of $E_{\mathrm{X}}$ ). This question will be addressed in more details in a further study, with a careful examination of the particle distributions.

Let us notice, for an possible application in the auroral context, that the growth of the plasma density perturbations

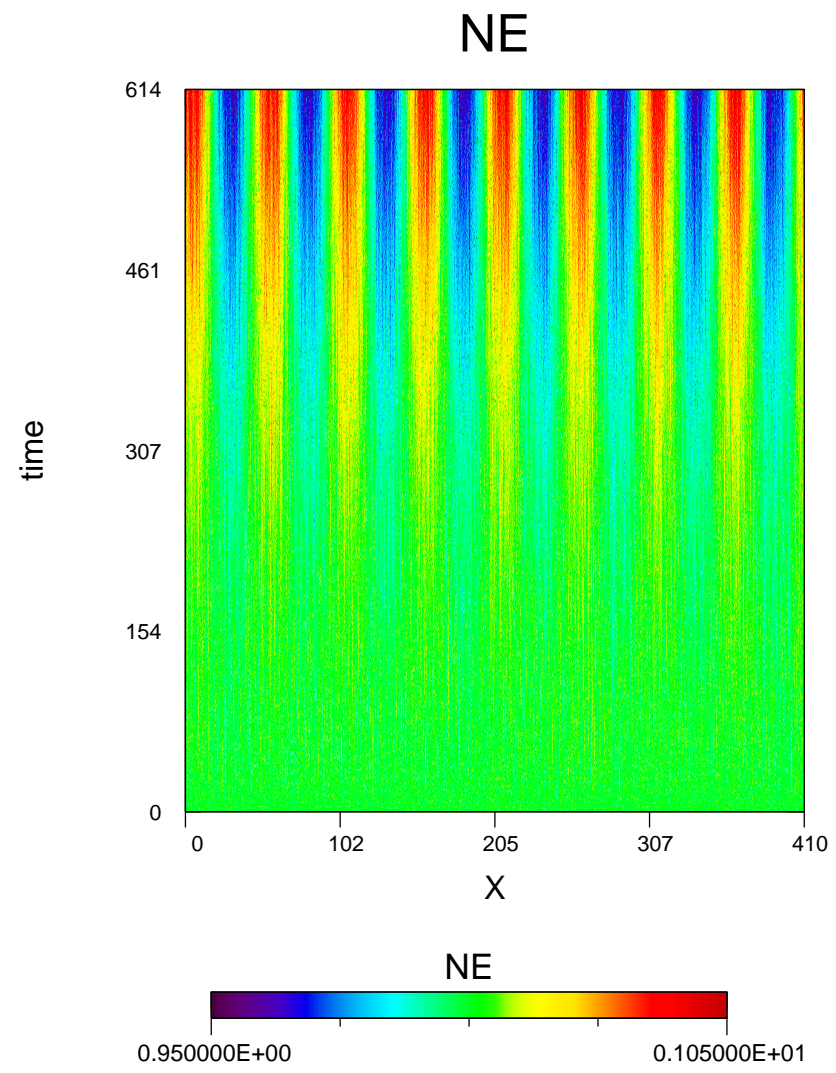

Fig. 9. AWC010. Temporal stack plot of the electron density $n_{\mathrm{e}}(X, t)$.

is not restricted to the case of sinusoidal waves. It happens with the crossing of two waves packets, and it can reach a very high amplitude, as shown in Fig. 10. In the auroral context, this modulation of the density would correspond to perturbations of the density as a function of the altitude, on a scale similar to those of the Alfvén wavelength.

\section{Conclusions}

This paper show a series of numerical experiments about the interaction of Alfvén waves propagating in opposite directions, in a situation that may be relevant for auroral physics.

It is shown, for the first time in the auroral context, that the interaction of counter-propagating Alfvén waves, with $k_{\perp}=0$, can generate longitudinal electric field structures. This process has been simulated with Alfvén wave packets and with Alfvén sinusoidal waves. The produced electric field structures do not propagate, provided that the locus of interaction of the two Alfvén waves is static.

Actually, a single wave packet of high intensity generates quasi-instantaneously a parallel electric field $E_{\mathrm{X}}$ of low amplitude. The role of the counter-propagating waves resides in the appearance of other space and time dependencies, 

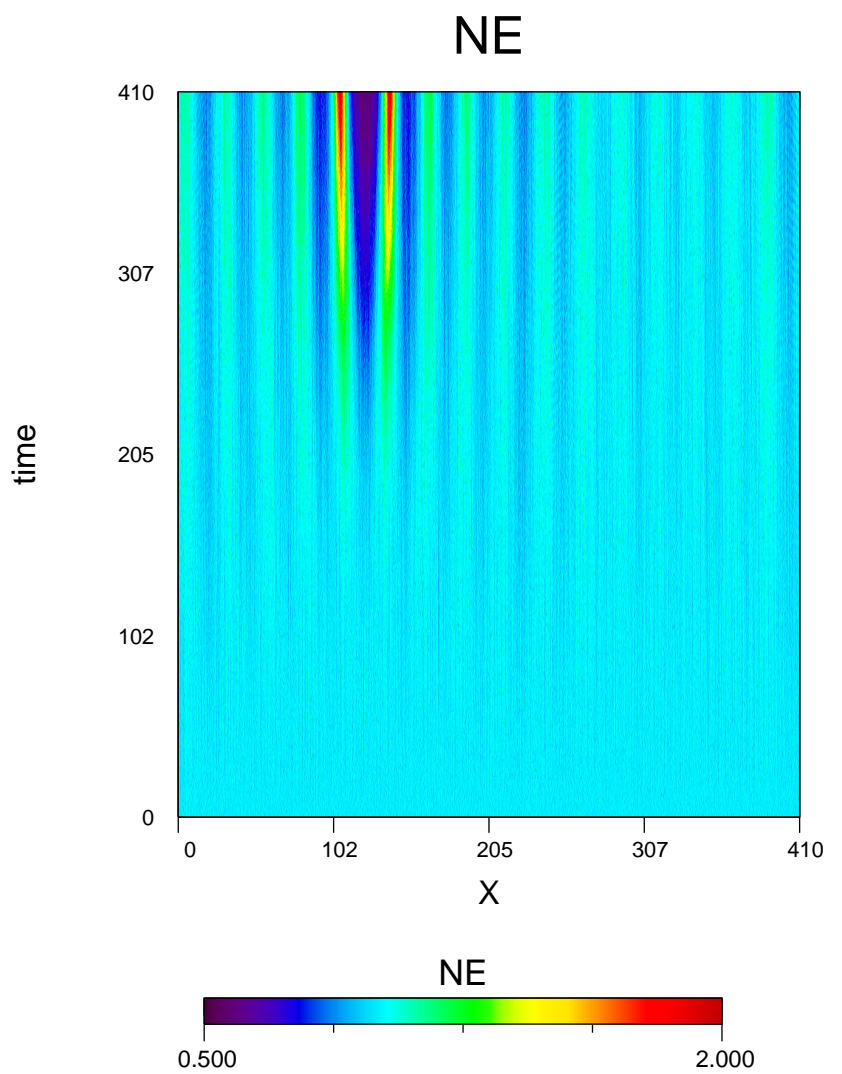

Fig. 10. AWC001. Temporal stack plot of the electron density $n_{\mathrm{e}}(X, t)$.

including the setting of a non-propagative structured longitudinal field $E_{\mathrm{X}}$.

Kraichnan (1965) showed that in incompressible MHD, the non-linear interactions occur only between Alfvén wave packets travelling in opposite directions. Therefore, the finite electric field $E_{\mathrm{X}}$ seen with waves propagating all in the same direction is the result of compressive or kinetic effects. It is suggested that the $E_{\mathrm{X}}$ electric component is generated by the distortion of the magnetic field lines by the Alfvén wave. Because of this distortion, the locally perpendicular electric field has a finite resulting projection along the $\mathrm{X}$-axis. This is a non-linear effect that has already been evidenced, with a single Alfvén wave, in the context of the solar corona physics by Tsiklauri (2006). It is associated to the growth of a variation, along the $\mathrm{X}$-axis, of the particle densities; it is therefore compressive.

The simulations conducted with two sinusoidal Alfvén waves show that the emergence of the electric field is quasiinstantaneous. At odds with the electric field, the growth of the density perturbation is progressive. The precise nature of the wave coupling associated to the emergence of the density perturbation and decay (Hung, 1974, parametric instability; Derby, 1978, effect of the accelerated particles?) has not been investigated yet.
The interactions observed in these simulations could be relevant for auroral physics. In that context, the two Alfvén waves would represent magnetic perturbations propagating downward, and reflected waves above the ionosphere. Therefore, we could expect, as in the present simulations, that the up- and down-going waves have roughly similar characteristics. Indeed, the amplitude of the reflected wave might be lower than that of the incident wave, but the simulations AWC007, AWC008, and AWC015-AWC019 with waves of different amplitudes have shown that this is not an obstacle to the growth the longitudinal electric field structures. These simulations are also associated to a growth of longitudinal density perturbations.

For computational reasons (to take profit of the guiding centre approximation for the electron motion), these simulations were conducted with an ambient magnetic field $B_{0}=4$ higher than in most regions of the auroral zone. Such high values (that correspond physically to a ratio of the electron gyrofrequency to the plasma frequency $\omega_{\mathrm{ce}} / \omega_{\mathrm{pe}}=4$ ) can be met only in the deep and localized plasma cavities with sharp transverse gradients observed in various acceleration regions (Hilgers et al., 1992; Delory et al., 1998; Chaston et al., 2007; Janhunen et al., 2004). These cavities are good places for electron acceleration by Alfvén waves (Génot et al., 2001b, 2004; Lysak and Song, 2008; Janhunen et al., 2004), but in the present case, a cavity is not required, and the phenomenon could happen in a denser, less magnetized homogeneous plasma. What would happen then? For the same Alfvén waves amplitude, the magnetic field line distortion would be larger. Therefore, the projection of the locally perpendicular electric field along the $\mathrm{X}$-axis would be larger too, and this would facilitate the creation of the $E_{\mathrm{X}}$ electric field. Therefore, the process could be even more efficient outside the auroral plasma cavities seen by Hilgers et al. (1992) and Delory et al. (1998).

Nevertheless, the problem is not completely solved. First, it is necessary to evaluate the influence of the parallel electric field on the particle distribution functions. It has been shown, in the framework of solar physics, that the interaction of sunward and anti-sunward Alfvén waves of similar amplitude through non-linear effects generates electrostatic waves and accelerate proton much more efficiently than in the quasilinear diffusion limit (Markovskii et al., 2009). Concerning auroral physics, does any Alfvén waves-electrostatic waves interaction accelerate the ions? Do they accelerate the electrons? Might mono-energetic beams be formed as with inverted V structures? These will be the topics of a forthcoming study.

Second, we can see in the simulations with wave packets (AWC001-AWC008) that the parallel electric field structures remain only during the interaction of the two Alfvén wave packets. As soon as the wave packets have crossed each other, the $E_{\mathrm{X}}$ structures starts to dissipate. Therefore, we have not yet created quasi-static inverted V-like structures that would survive the Alfvénic acceleration phase (apart 
from the case of AWC002 where it is not yet possible to conclude). In order to make quasi-static structures, one could imagine that in the ionospheric Alfvénic resonator (Chaston et al., 2002), there would be a quasi-continuous supply of incident and reflected waves, and that these electric field structures would develop at the antinodes whose existence would be almost permanent. On the contrary, the density structures do not disappear after the wave crossing. As can be seen on Fig. 10, the density perturbation continues to grow after the wave packets crossing.

The non-linear coupling of counter-propagating Alfvén waves trapped in an Earth auroral resonant cavity has been investigated by Chian (1995). Although they did not consider the emergence of a longitudinal electric field, they have shown that electrostatic waves can be generated in that context. In the present study, we show also that these waves can be the cause of non-propagating density structures.

The projection effect invoked in Sect. 4 has already been studied in a work by Knudsen (1996) concerning the auroral context: there were up and down going Alfvén waves, but with a slightly oblique direction of propagation, implying electron inertia effects. The projection effect that was analysed depended rather on the coupling of each of these waves with the permanent electric field associated to a field aligned current sheet. A modulation of the plasma density was observed, as in the present paper. Low density cavities can also be created by ion heating localized in a region of a few gyroradius transverse size. Then, the process is associated to the growth of lower-hybrid waves and to the ponderomotive force. It can create small size density cavities (Seyler, 1994; Knudsen et al., 2004). This effect can be amplified by the action of the mirror force caused by the convergence of the magnetic field lines in the direction of the Earth (Singh, 1994). Other ways of initiating plasma density variations have been elaborated in the auroral context. The ponderomotive force associated to the coupling of an Alfvén wave and an ion-acoustic wave could create deep density cavities (Sharma and Singh, 2009). On a larger scale, the growth of density variations is associated to the ionospheric feedback instability, which is a coupling of Alfvén waves with the ionosphere (Streltsov and Lotko, 2008). In that case, the density cavities are not created at magnetospheric altitudes, but in the bottomside ionosphere, a region that can be observed with ground based radars. Still on a large scale, the ponderomotive force associated to standing oscillations in the ionospheric Alfvénic resonator can create plasma flows along the geomagnetic field that modify the plasma density profile (Sydorenko et al., 2008).

At odds with the above cited works, the present study is based only on the interaction of Alfvén waves above the ionosphere, it does not require the presence of an electric current or a large scale background density gradient, and it does not depend on the mirror force. It can be complementary to the previous studies, and especially relevant to cavities formed inside the auroral Alfvénic resonator.
The simultaneous presence of Alfvénic acceleration and quasi-static acceleration structures has recently been diagnosed outside the Earth auroral zone, in the magnetic flux tube connecting the planet Jupiter to its satellite Io. The diagnostics were made through the analysis of the so-called S-bursts and N-band radio emissions (Hess et al., 2009b; Arkhypov and Rucker, 2011) and a combination of data analysis and numerical simulation (Hess et al., 2007, 2009a). In a discussion paper, it has been established that the observed quasi-static structures are below the Alfvénic acceleration region, but still in the Alfvénic resonator, in the place where both the incident and the reflected Alfvén waves have a high amplitude (Mottez et al., 2010). Although we have no proof yet that the Alfvénic acceleration would precede the formation of quasi-static acceleration structures, the mechanism presented in this paper might be interesting in the Jovian case too.

Acknowledgements. The author thanks Vincent Génot for useful discussions. The numerical simulations were performed at the computing center (DIO) of the Paris-Meudon observatory.

Topical Editor R. Nakamura thanks two anonymous referees for their help in evaluating this paper.

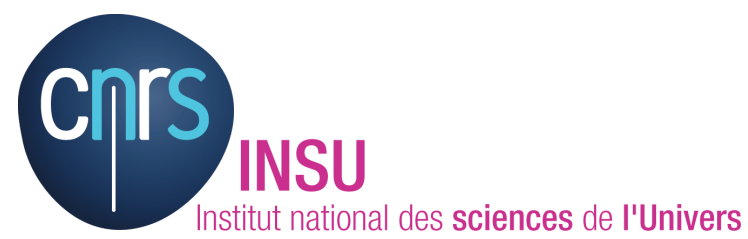

The publication of this article is financed by CNRS-INSU.

\section{References}

Arkhypov, O. V. and Rucker, H. O.: Dynamics of decametric radiosources and standing Alfvén waves in jovian magnetosphere, Icarus, 212, 714-718, doi:10.1016/j.icarus.2011.01.009, 2011.

Asamura, K., Chaston, C. C., Itoh, Y., Fujimoto, M., Sakanoi, T., Ebihara, Y., Yamazaki, A., Hirahara, M., Seki, K., Kasaba, Y., and Okada, M.: Sheared flows and small-scale Alfvén wave generation in the auroral acceleration region, Geophys. Res. Lett., 36, 5105, doi:10.1029/2008GL036803, 2009.

Chaston, C. C., Bonnell, J. W., Carlson, C. W., Berthomier, M., Peticolas, L. M., Roth, I., McFadden, J. P., Ergun, R. E., and Strangeway, R. J.: Electron acceleration in the ionospheric Alfven resonator, J. Geophys. Res. (Space Physics), 107, 41-1, doi:10.1029/2002JA009272, 2002.

Chaston, C. C., Hull, A. J., Bonnell, J. W., Carlson, C. W., Ergun, R. E., Strangeway, R. J., and McFadden, J. P.: Large parallel electric fields, currents, and density cavities in dispersive Alfvén waves above the aurora, J. Geophys. Res. (Space Physics), 112, A05215, doi:10.1029/2006JA012007, 2007.

Chen, L., Kletzing, C. A., Hu, S., and Bounds, S. R.: Auroral electron dispersion below inverted-V energies: Resonant deceleration and acceleration by Alfvén waves, J. Geophys. Res. (Space Physics), 110, 10, doi:10.1029/2005JA011168, 2005. 
Chian, A.: Nonlinear Alfvén wave phenomena in the planetary magnetosphere, Physica Scripta Volume T, 60, 36-43, doi:10.1088/0031-8949/1995/T60/005, 1995.

Chust, T., Louarn, P., Volwerk, M., de Feraudy, H., Roux, A., Wahlund, J., and Holback, B.: Electric fields with a large parallel component observed by the Freja spacecraft: Artifacts or real signals?, J. Geophys. Res. (Space Physics), 103, 215-224, doi:10.1029/97JA02587, 1998.

Delory, G. T., Ergun, R. E., Carlson, C. W., Muschietti, L., Chaston, C. C., Peria, W., McFadden, J. P., and Strangeway, R.: FAST observations of electron distributions within AKR source regions, Geophys. Res. Lett., 25, 2069-2072, doi:10.1029/98GL00705, 1998.

Derby, Jr., N. F.: Modulational instability of finite-amplitude, circularly polarized Alfven waves, Astrophys. J., 224, 1013-1016, doi:10.1086/156451, 1978.

Ergun, R. E., Andersson, L., Su, Y.-J., Newman, D. L., Goldman, M. V., Lotko, W., Chaston, C. C., and Carlson, C. W.: Localized parallel electric fields associated with inertial Alfvén waves, Physics of Plasmas, 12, 072901, doi:10.1063/1.1924495, 2005.

Frey, H. U., Amm, O., Chaston, C. C., Fu, S., Haerendel, G., Juusola, L., Karlsson, T., Lanchester, B., Nakamura, R., Østgaard, N., Sakanoi, T., Séran, E., Whiter, D., Weygand, J., Asamura, K., and Hirahara, M.: Small and meso-scale properties of a substorm onset auroral arc, J. Geophys. Res. (Space Physics), 115, 10209, doi:10.1029/2010JA015537, 2010.

Génot, V., Louarn, P., and Mottez, F.: Electron acceleration by Alfvén waves in density cavities, J. Geophys. Res. (Space Physics), 105, 27611-27620, doi:10.1029/1999JA000341, 2000.

Génot, V., Louarn, P., and Mottez, F.: Fast evolving spatial structure of auroral parallel electric fields, J. Geophys. Res., 106, 2963329644, 2001a.

Génot, V., Mottez, F., and Louarn, P.: Particle Acceleration Linked to Alfven Wave Propagation on Small Scale Density Gradients, Phys. Chem. Earth C, 26, 219-222, doi:10.1016/S14641917(00)00111-2, 2001 b.

Génot, V., Louarn, P., and Mottez, F.: Alfvén wave interaction with inhomogeneous plasmas: acceleration and energy cascade towards small-scales, Ann. Geophys., 22, 2081-2096, doi:10.5194/angeo-22-2081-2004, 2004.

Goertz, C. K.: Kinetic Alfvén waves on auroral field lines, Planet. Space Sci., 32, 1387-1392, 1984.

Hasegawa, A. and Chen, L.: Kinetic process of plasma heating due to Alfvén wave excitation, Phys. Rev. Lett., 35, 370-373, 1975.

Hess, S., Mottez, F., and Zarka, P.: Jovian S burst generation by Alfvén waves, J. Geophys. Res. (Space Physics), 112, 11212, doi:10.1029/2006JA012191, 2007.

Hess, S., Mottez, F., and Zarka, P.: Effect of electric potential structures on Jovian S-burst morphology, Geophys. Res. Lett., 36, 14101, doi:10.1029/2009GL039084, 2009a.

Hess, S., Zarka, P., Mottez, F., and Ryabov, V. B.: Electric potential jumps in the Io-Jupiter flux tube, Planet. Space Sci., 57, 23-33, doi:10.1016/j.pss.2008.10.006, 2009b.

Hilgers, A., Holback, B., Holmgren, G., and Bostrom, R.: Probe measurements of low plasma densities with applications to the auroral acceleration region and auroral kilometric radiation sources, J. Geophys. Res. (Space Physics), 97, 8631-8641, doi:10.1029/91JA02193, 1992.

Hull, A. J., Wilber, M., Chaston, C. C., Bonnell, J. W., Mc-
Fadden, J. P., Mozer, F. S., Fillingim, M., and Goldstein, M. L.: Time development of field-aligned currents, potential drops, and plasma associated with an auroral poleward boundary intensification, J. Geophys. Res. (Space Physics), 115, 6211, doi:10.1029/2009JA014651, 2010.

Hung, N. T.: Parametric excitation of Alfven and acoustic waves, J. Plasma Physics, 12, 445-453, doi:10.1017/S0022377800025393, 1974.

Janhunen, P., Olsson, A., Hanasz, J., Russell, C. T., Laakso, H., and Samson, J. C.: Different Alfvén wave acceleration processes of electrons in substorms at $\sim 4-5 R_{\mathrm{E}}$ and $2-3 R_{\mathrm{E}}$ radial distance, Ann. Geophys., 22, 2213-2227, doi:10.5194/angeo-222213-2004, 2004.

Keiling, A.: Alfvén Waves and Their Roles in the Dynamics of the Earth's Magnetotail: A Review, Space Sci. Rev., 142, 73-156, doi:10.1007/s11214-008-9463-8, 2009.

Keiling, A., Wygant, J. R., Cattell, C., Temerin, M., Mozer, F. S., Kletzing, C. A., Scudder, J., Russell, C. T., Lotko, W., and Streltsov, A. V.: Large Alfvén wave power in the plasma sheet boundary layer during the expansion phase of substorms, Geophys. Res. Lett., 27, 3169-3172, doi:10.1029/2000GL000127, 2000.

Klatt, E. M., Kintner, P. M., Seyler, C. E., Liu, K., MacDonald, E. A., and Lynch, K. A.: SIERRA observations of Alfvénic processes in the topside auroral ionosphere, J. Geophys. Res. (Space Physics), 110, 10, doi:10.1029/2004JA010883, 2005.

Knudsen, D. J.: Spatial modulation of electron energy and density by nonlinear stationary inertial Alfvén waves, J. Geophys. Res. (Space Physics), 101, 10761-10772, doi:10.1029/96JA00429, 1996.

Knudsen, D. J., Bock, B. J. J., Bounds, S. R., Burchill, J. K., Clemmons, J. H., Curtis, J. D., Eriksson, A. I., Koepke, M. E., Pfaff, R. F., Wallis, D. D., and Whaley, N.: Lower-hybrid cavity density depletions as a result of transverse ion acceleration localized on the gyroradius scale, J. Geophys. Res. (Space Physics), 109, 4212, doi:10.1029/2003JA010089, 2004.

Kraichnan, R. H.: Inertial-Range Spectrum of Hydromagnetic Turbulence, Physics of Fluids, 8, 1385-1387, doi:10.1063/1.1761412, 1965.

Lessard, M. R. and Knudsen, D. J.: Ionospheric reflection of small-scale Alfvén waves, Geophys. Res. Lett., 28, 3573-3576, doi:10.1029/2000GL012529, 2001.

Louarn, P., Wahlund, J. E., Chust, T., de Feraudy, H., Roux, A., Holback, B., Dovner, P. O., Eriksson, A. I., and Holmgren, G.: Observation of kinetic Alfven waves by the Freja spacecraft, Geophys. Res. Lett., 21, 1847, doi:10.1029/94GL00882, 1994.

Lysak, R. L. and Song, Y.: Propagation of kinetic Alfvén waves in the ionospheric Alfvén resonator in the presence of density cavities, Geophys. Res. Lett., 35, 20101, doi:10.1029/2008GL035728, 2008.

Markovskii, S. A., Vasquez, B. J., and Hollweg, J. V.: Proton Heating by Nonlinear Field-Aligned Alfvén Waves in Solar Coronal Holes, The Astrophysical Journal, 695, 1413-1420, doi:10.1088/0004-637X/695/2/1413, 2009.

Mottez, F.: A guiding centre direct implicit scheme for magnetized plasma simulations, J. Comput. Phys., 227, 3260-3281, doi:10.1016/j.jcp.2007.11.034, 2008.

Mottez, F. and Génot: Electron acceleration by an Alfvnic pulse propagating in an auroral plasma cavity, J. Geophys. Res. (Space 
Physics), 116, A00K15, doi:10.1029/2010JA016367, 2011.

Mottez, F., Adam, J. C., and Heron, A.: A new guiding centre PIC scheme for electromagnetic highly magnetized plasma simulation, Computer Physics Communications, 113, 109-130, doi:10.1016/S0010-4655(98)00076-9, 1998.

Mottez, F., Hess, S., and Zarka, P.: Explanation of dominant oblique radio emission at Jupiter and comparison to the terrestrial case, Planet. Space Sci., 58, 1414-1422, doi:10.1016/j.pss.2010.05.012, 2010.

Newell, P. T., Lee, A. R., Liou, K., Ohtani, S., Sotirelis, T., and Wing, S.: Substorm cycle dependence of various types of aurora, J. Geophys. Res. (Space Physics), 115, 9226, doi:10.1029/2010JA015331, 2010.

Passot, T. and Vázquez-Semadeni, E.: The correlation between magnetic pressure and density in compressible MHD turbulence, Astronomy and Astrophysics, 398, 845-855, doi:10.1051/00046361:20021665, 2003.

Seyler, C. E.: Lower hybrid wave phenomena associated with density depletions, J. Geophys. Res. (Space Physics), 99, 19513, doi:10.1029/94JA01572, 1994.

Sharma, R. P. and Singh, H. D.: Density cavities associated with inertial Alfvén waves in the auroral plasma, J. Geophys. Res. (Space Physics), 114, 3109, doi:10.1029/2008JA013474, 2009.

Singh, N.: Pondermotive versus mirror force in creation of the filamentary cavities in auroral plasma, Geophys. Res. Lett., 21, 257260, doi:10.1029/93GL03387, 1994.
Streltsov, A. V. and Lotko, W.: Coupling between density structures, electromagnetic waves and ionospheric feedback in the auroral zone, J. Geophys. Res. (Space Physics), 113, 5212, doi:10.1029/2007JA012594, 2008.

Sydorenko, D., Rankin, R., and Kabin, K.: Nonlinear effects in the ionospheric Alfvén resonator, J. Geophys. Res. (Space Physics), 113, 10206, doi:10.1029/2008JA013579, 2008.

Tsiklauri, D.: A mechanism for parallel electric field generation in the MHD limit: possible implications for the coronal heating problem in the two stage mechanism, Astronomy and Astrophysics, 455, 1073-1080, doi:10.1051/0004-6361:20064816, 2006.

Volwerk, M., Louarn, P., Chust, T., Roux, A., de Feraudy, H., and Holback, B.: Solitary kinetic Alfvén waves: A study of the Poynting flux, J. Geophys. Res. (Space Physics), 101, 1333513344, doi:10.1029/96JA00166, 1996.

Zou, S., Moldwin, M. B., Lyons, L. R., Nishimura, Y., Hirahara, M., Sakanoi, T., Asamura, K., Nicolls, M. J., Miyashita, Y., Mende, S. B., and Heinselman, C. J.: Identification of substorm onset location and preonset sequence using Reimei, THEMIS GBO, PFISR, and Geotail, J. Geophys. Res. (Space Physics), 115, 12309, doi:10.1029/2010JA015520, 2010. 